\title{
Regularity results and asymptotic behavior for a noncoercive parabolic problem
}

\author{
Lucio Boccardo, Luigi Orsina(D AND Maria Michaela Porzio
}

Abstract. In this paper we study the regularity and the behavior in time of the solutions to a quasilinear class of noncoercive problems whose prototype is

$$
\begin{cases}u_{t}-\operatorname{div}(a(x, t, u) \nabla u)=-\operatorname{div}(u E(x, t)) & \text { in } \Omega \times(0, T), \\ u(x, t)=0 & \text { on } \partial \Omega \times(0, T), \\ u(x, 0)=u_{0}(x) & \text { in } \Omega .\end{cases}
$$

In particular we show that under suitable conditions on the vector field $E$, even if the problem is noncoercive and although the initial datum $u_{0}$ is only an $L^{1}(\Omega)$ function, there exist solutions that immediately improve their regularity and belong to every Lebesgue space. We also prove that solutions may become immediately bounded. Finally, we study the behavior in time of such regular solutions and we prove estimates that allow to describe their blow-up for $t$ near zero.

\section{Introduction}

Let us consider the following parabolic problem

$$
\begin{cases}u_{t}(x, t)-\operatorname{div}(a(x, t, u(x, t)) \nabla u(x, t))=-\operatorname{div}(u(x, t) E(x, t)) & \text { in } Q_{T} \equiv \Omega \times(0, T), \\ u(x, t)=0 & \text { on } \partial \Omega \times(0, T), \\ u(x, 0)=u_{0}(x) & \text { in } \Omega,\end{cases}
$$

where $\Omega$ is an open bounded subset of $\mathbb{R}^{N}, N>2$, and $T>0$. We assume that $a(x, t, s): Q_{T} \times \mathbb{R} \rightarrow \mathbb{R}$ is a Carathéodory function (i.e., measurable in $(x, t)$ for every $s$ in $\mathbb{R}$, and continuous in $s$ for almost every $(x, t)$ in $\left.Q_{T}\right)$ such that

$$
0<\alpha \leq a(x, t, s) \leq \beta
$$

for almost every $(x, t)$ in $Q_{T}$ and for every $s$ in $\mathbb{R}$, where $\alpha$ and $\beta$ are positive constants. On the initial datum we assume

$$
u_{0} \in L^{1}(\Omega)
$$

Keywords: Linear and quasilinear parabolic equations, Asymptotic behavior, Regularity of solutions, Noncoercive problems. 
The main difficulty in studying this problem is the presence of the lower order term $-\operatorname{div}(u E(x, t))$ which makes the problem noncoercive. In addition, the initial datum has a very low regularity, so that the study of such an equation is more complicated.

In the stationary case this problem was studied in the sixties by Stampacchia (see $[14,15])$ when $E$ "is not too large", and then by several authors until nowadays (see for example $[2,3,6,8]$ and the references therein).

In the evolution case existence and regularity results can be found in [4] (see also [9]).

We recall that if $E$ is zero then a very surprising phenomenon appears: even if $u_{0}$ is only a summable function, there exists a solution $u$ of (1.1) that becomes immediately (i.e., for every $t>0$ ) bounded and satisfies the same decay (or ultracontractive estimate) of the solution of the heat equation, i.e.,

$$
\|u(t)\|_{L^{\infty}(\Omega)} \leq C(N, \alpha) \frac{\left\|u_{0}\right\|_{L^{1}(\Omega)}}{t^{\frac{N}{2}}} \text { for every } t \in(0, T),
$$

(see $[5,12,16])$.

The aim of this paper is to understand what happens when $E$ is a nonzero measurable vector field; in particular, we want to understand if an immediate improvement in the regularity of the solutions appears or not. We will prove (see Sect. 2) that if $E$ satisfies

$$
|E(x, t)| \leq \gamma|B(x)| \quad B \in L^{N}(\Omega),
$$

for almost every $(x, t)$ in $Q_{T}$, then there is an immediate regularization since there exists a solution $u$ of (1.1) which belongs to $L^{\infty}\left(t_{0}, T ; L^{q}(\Omega)\right)$ for every $1<q<+\infty$ and $t_{0}$ in $(0, T)$. Besides, we will derive quantitative estimates "near $t=0$ " (see Sect. 2.1) and for $t$ large (see Sect. 2.2).

Moreover, we show that under stronger assumptions on $E$ solutions become immediately bounded (see Sect. 3); furthermore, near " $t=0$ " the same behavior (1.4) of the solution of the heat equation holds true, but in this case the constant $c$ in (1.4) depends also on $E$.

Finally, in Sect. 4 we study the less regular case of a vector field $E$ such that

$$
|E(x, t)| \leq \frac{\gamma}{|x|}, \quad \gamma>0,
$$

which may not belong to $L^{\infty}\left(0, T ; L^{N}(\Omega)\right)$ if 0 belongs to $\Omega$.

Remark 1.1. We would like to thank the referee of the paper for pointing out that in the autonomous case, i.e., for the equation

$$
u_{t}-\operatorname{div}(A(x) \nabla u)=-\operatorname{div}(u E(x)),
$$

with $E$ in $L^{\infty}(\Omega)$, the following holds:

- the operator

$$
A(u)=-\operatorname{div}(A(x) \nabla u)+\operatorname{div}(u E(x))
$$


is quasi accretive in $L^{q}(\Omega)$ spaces (for every $1 \leq q \leq \infty$ ), and quasi $m$-accretive in $L^{q}(\Omega)$ spaces (for every $1 \leq q<\infty$ );

- every mild solution $u$ of (1.6) satisfies an $L^{1}-L^{\infty}$ estimate similar to (1.4) thanks to Theorem 1.2 of [7].

\section{An improvement of regularity}

In this section we will show that even if the initial datum $u_{0}$ is only assumed to be a summable function, if $E$ satisfies (1.5) there exists a solution of (1.1) which belongs to every Lebesgue space $L^{\infty}\left(t_{0}, T ; L^{q}(\Omega)\right)$ for every $1<q<+\infty$ and $t_{0} \in(0, T)$ (see Theorem 2.1 in Sect. 2.1 below). Moreover, we describe the blow-up of the $L^{q}(\Omega)$-norm of $u(t)$ as $t$ tends to zero and the behavior of the solution for $t$ large.

We recall that here by a solution of (1.1) we mean a function $u$ in $L^{\infty}\left(0, T ; L^{1}(\Omega)\right)$ and $L^{1}\left(0, T ; W_{0}^{1,1}(\Omega)\right)$ such that

$$
\int_{Q_{T}}\left[-u \varphi_{t}+a(x, t, u) \nabla u \nabla \varphi\right]=\int_{Q_{T}} u E \nabla \varphi+\int_{\Omega} u_{0} \varphi(0),
$$

for every $\varphi$ in $W^{1, \infty}\left(0, T ; L^{\infty}(\Omega)\right) \cap L^{\infty}\left(0, T ; W_{0}^{1, \infty}(\Omega)\right)$ such that $\varphi(T)=0$.

We recall that, thanks to the results of [4], under assumptions (1.3) and (1.5) there exists a solution $u$ of $(1.1)$, with $u$ in $L^{\infty}\left(0, T ; L^{1}(\Omega)\right) \cap L^{q}\left(0, T ; W_{0}^{1, q}(\Omega)\right)$, with $q=\frac{N+2}{N+1}$ (see Lemma 3.2 of [4]). Furthermore, if $|E|$ belongs to $L^{\infty}\left(Q_{T}\right)$ and $u_{0}$ belongs to $L^{\infty}(\Omega)$, there exists a bounded solution $u$ of (1.1) such that (see Lemma 3.1 of [4])

$$
\|u(t)\|_{L^{1}(\Omega)} \leq\left\|u_{0}\right\|_{L^{1}(\Omega)} \quad \text { for almost every } t \in(0, T) .
$$

\subsection{Behaviour near zero}

Theorem 2.1. Assume (1.2), (1.3) and (1.5). Then there exists a solution $u$ of (1.1) satisfying

$$
u \in L^{\infty}\left(t_{0}, T ; L^{q}(\Omega)\right) \text { for every } t_{0} \text { in }(0, T) \text {, and for every } 1<q<+\infty .
$$

Furthermore, the following estimate holds:

$$
\|u\|_{L^{\infty}\left(t_{0}, T ; L^{q}(\Omega)\right)} \leq c_{0}\left\|u_{0}\right\|_{L^{1}(\Omega)}
$$

where $c_{0}$ is a constant depending only on $t_{0}, q, N, \alpha, T$ and $B$. Moreover, for every $q \geq 2$ and $t_{0}>0$ it results

$$
\left.|\nabla| u\right|^{\frac{q}{2}-1} u\left|\in L^{2}\left(t_{0}, T ; L^{2}(\Omega)\right), \quad\right| \nabla u \mid \in L^{2}\left(t_{0}, T ; L^{2}(\Omega)\right) .
$$


Finally,

$$
\|u(t)\|_{L^{q}(\Omega)} \leq c_{1} \frac{\left\|u_{0}\right\|_{L^{1}(\Omega)}}{t^{\frac{N}{2}\left(1-\frac{1}{q}\right)}} \quad \text { for almost every } t \text { in }(0, T),
$$

where $c_{1}$ is a constant depending only on $N, q, \alpha, \gamma, T$ and B (see formula (2.21)).

Proof. In [4] it is proved the existence of a solution $u$ of (1.1), obtained as the almost everywhere limit of a sequence of weak solutions $u_{n}$ in $L^{2}\left(0, T ; W_{0}^{1,2}(\Omega)\right) \cap$ $C\left([0, T] ; L^{2}(\Omega)\right)$ of the following problems:

$$
\begin{cases}\left(u_{n}\right)_{t}-\operatorname{div}\left(a\left(x, t, u_{n}\right) \nabla u_{n}\right)=-\operatorname{div}\left(T_{n}\left(u_{n}\right) E_{n}(x, t)\right) & \text { in } Q_{T} \equiv \Omega \times(0, T), \\ u_{n}(x, t)=0 & \text { on } \partial \Omega \times(0, T), \\ u_{n}(x, 0)=T_{n}\left(u_{0}(x)\right) & \text { in } \Omega,\end{cases}
$$

where $T_{n}$ is the usual truncating function

$$
T_{n}(s)=\min \{n, \max \{-n, s\}\}, \quad s \in \mathbb{R},
$$

and

$$
E_{n}(x, t)=\frac{E(x, t)}{1+\frac{1}{n}|E(x, t)|} .
$$

Notice that since $T_{n}\left(u_{n}\right) E_{n}(x, t)$ belongs to $L^{\infty}\left(Q_{T}\right)$ then $u_{n}$ also belongs $L^{\infty}\left(Q_{T}\right)$ by the results of [1]. We fix $q>1, t>0$, choose as test function in (2.5) $v_{n}=$ $\left|u_{n}\right|^{q-2} u_{n}$, and integrate on $\Omega$. We obtain, using (1.2), and the fact that $\left|T_{n}(s)\right| \leq|s|$,

$$
\begin{aligned}
\frac{1}{q} \frac{d}{d t} & \int_{\Omega}\left|u_{n}(t)\right|^{q}+\alpha(q-1) \int_{\Omega}\left|\nabla u_{n}(t)\right|^{2}\left|u_{n}(t)\right|^{q-2} \\
& \leq(q-1) \int_{\Omega} T_{n}\left(u_{n}(t)\right) E_{n} \cdot \nabla u_{n}(t)\left|u_{n}(t)\right|^{q-2} \\
& \leq(q-1) \int_{\Omega}|E|\left|\nabla u_{n}(t)\right|\left|u_{n}(t)\right|^{q-1} .
\end{aligned}
$$

We now work with the right hand side; using Young inequality, as well as (1.5), we have

$$
\begin{aligned}
\int_{\Omega}|E|\left|\nabla u_{n}(t)\right|\left|u_{n}(t)\right|^{q-1} & \leq \gamma \int_{\Omega}\left|\nabla u_{n}(t)\right||B(x)|\left|u_{n}(t)\right|^{q-1} \\
& \leq \frac{\alpha}{2} \int_{\Omega}\left|\nabla u_{n}(t)\right|^{2}\left|u_{n}(t)\right|^{q-2}+C_{1} \int_{\Omega}|B(x)|^{2}\left|u_{n}(t)\right|^{q},
\end{aligned}
$$

where $C_{1}=\frac{\gamma^{2}}{2 \alpha}$. Therefore, part of the right hand side can be absorbed in the left hand one, to obtain

$$
\frac{1}{q} \frac{d}{d t} \int_{\Omega}\left|u_{n}(t)\right|^{q}+\frac{\alpha(q-1)}{2} \int_{\Omega}\left|\nabla u_{n}(t)\right|^{2}\left|u_{n}(t)\right|^{q-2} \leq C_{2} \int_{\Omega}|B(x)|^{2}\left|u_{n}(t)\right|^{q},
$$


where $C_{2}=C_{2}(q)=C_{1}(q-1)$. We continue to work on the right hand side; we have, for some $\rho>0$, and thanks to Hölder inequality,

$$
\begin{aligned}
& \int_{\Omega}|B(x)|^{2}\left|u_{n}(t)\right|^{q}=\int_{\{|B(x)| \leq \rho\}}|B(x)|^{2}\left|u_{n}(t)\right|^{q}+\int_{\{|B(x)|>\rho\}}|B(x)|^{2}\left|u_{n}(t)\right|^{q} \\
& \leq \rho^{2} \int_{\Omega}\left|u_{n}(t)\right|^{q}+\int_{\{|B(x)|>\rho\}}|B(x)|^{2}\left|u_{n}(t)\right|^{q} \\
& \leq \rho^{2} \int_{\Omega}\left|u_{n}(t)\right|^{q}+\left(\int_{\{|B(x)|>\rho\}}|B(x)|^{N}\right)^{\frac{2}{N}}\left(\int_{\Omega}\left|u_{n}(t)\right|^{\frac{2^{*}}{2} q}\right)^{\frac{2}{2^{*}}}
\end{aligned}
$$

where, as usual, $2^{*}=\frac{2 N}{N-2}$ is the Sobolev embedding exponent. Since, by Sobolev embedding, we have

$$
\left(\int_{\Omega}\left|u_{n}(t)\right|^{2^{*} \frac{q}{2}}\right)^{\frac{2}{2^{*}}} \leq \mathcal{S} \int_{\Omega}\left|\nabla\left[\left|u_{n}(t)\right|^{\frac{q}{2}} \operatorname{sign}\left(u_{n}\right)\right]\right|^{2}=\frac{q^{2} \mathcal{S}}{4} \int_{\Omega}\left|\nabla u_{n}(t)\right|^{2}\left|u_{n}(t)\right|^{q-2},
$$

we therefore have that

$$
\begin{aligned}
& C_{2} \int_{\Omega}|B(x)|^{2}\left|u_{n}(t)\right|^{q} \leq C_{2} \rho^{2} \int_{\Omega}\left|u_{n}(t)\right|^{q} \\
& +C_{2} \frac{q^{2} \mathcal{S}}{4}\left(\int_{\{|B(x)|>\rho\}}|B(x)|^{N}\right)^{\frac{2}{N}} \int_{\Omega}\left|\nabla u_{n}(t)\right|^{2}\left|u_{n}(t)\right|^{q-2} .
\end{aligned}
$$

We now choose $\rho$ in such a way that

$$
C_{2} \frac{q^{2} \mathcal{S}}{4}\left(\int_{\{|B(x)|>\rho\}}|B(x)|^{N}\right)^{\frac{2}{N}} \leq \frac{\alpha(q-1)}{4} .
$$

Such a choice is possible since $B$ belongs to $L^{N}(\Omega)$. Note that $\rho$ does not depend on $t$. We therefore have that

$$
C_{2} \int_{\Omega}|B(x)|^{2}\left|u_{n}(t)\right|^{q} \leq C_{3} \int_{\Omega}\left|u_{n}(t)\right|^{q}+\frac{\alpha(q-1)}{4} \int_{\Omega}\left|\nabla u_{n}(t)\right|^{2}\left|u_{n}(t)\right|^{q-2},
$$

where $C_{3}$ is a constant depending only on $\alpha, q, \gamma, \mathcal{S}$ and $B$. Substituting this inequality in (2.8) we obtain, after simplifying equal terms,

$$
\frac{1}{q} \frac{d}{d t} \int_{\Omega}\left|u_{n}(t)\right|^{q}+\frac{\alpha(q-1)}{4} \int_{\Omega}\left|\nabla u_{n}(t)\right|^{2}\left|u_{n}(t)\right|^{q-2} \leq C_{3} \int_{\Omega}\left|u_{n}(t)\right|^{q} .
$$

Writing $\left|\nabla u_{n}(t)\right|^{2}\left|u_{n}(t)\right|^{q-2}=\frac{4}{q^{2}}\left|\nabla\left[\left|u_{n}(t)\right|^{\frac{q}{2}} \operatorname{sign}\left(u_{n}\right)\right]\right|^{2}$, and using Sobolev embedding, we arrive at

$$
\frac{1}{q} \frac{d}{d t} \int_{\Omega}\left|u_{n}(t)\right|^{q}+\frac{\alpha(q-1)}{q^{2} \mathcal{S}}\left(\int_{\Omega}\left|u_{n}(t)\right|^{\frac{2^{*}}{2} q}\right)^{\frac{2}{2^{*}}} \leq C_{3} \int_{\Omega}\left|u_{n}(t)\right|^{q} .
$$


Since $1<q<\frac{2^{*}}{2} q$, we can interpolate, to obtain

$$
\int_{\Omega}\left|u_{n}(t)\right|^{q} \leq\left(\int_{\Omega}\left|u_{n}(t)\right|\right)^{q(1-\theta)}\left(\int_{\Omega}\left|u_{n}(t)\right|^{\frac{2^{*}}{2} q}\right)^{\frac{2}{2^{*}} \theta},
$$

where $\theta$ in $(0,1)$ is such that

$$
\frac{1}{q}=(1-\theta) \frac{1}{1}+\theta \frac{2}{2 * q} .
$$

We recall now (see the proof of Lemma 3.1 in [4]) that (2.1) holds true for $u_{n}$, so that

$$
\left\|u_{n}(t)\right\|_{L^{1}(\Omega)} \leq\left\|T_{n}\left(u_{0}\right)\right\|_{L^{1}(\Omega)}, \quad \text { for every } t \text { in }(0, T) .
$$

Hence, from (2.14) it follows that

$$
\begin{aligned}
& \int_{\Omega}\left|u_{n}(t)\right|^{q} \leq\left(\int_{\Omega}\left|T_{n}\left(u_{0}\right)\right|\right)^{q(1-\theta)}\left(\int_{\Omega}\left|u_{n}(t)\right|^{\frac{2^{*}}{2} q}\right)^{\frac{2}{2^{*}} \theta} \\
& \leq\left(\int_{\Omega}\left|u_{0}\right|\right)^{q(1-\theta)}\left(\int_{\Omega}\left|u_{n}(t)\right|^{\frac{2^{*}}{2}} q\right)^{\frac{2}{2^{*}} \theta} .
\end{aligned}
$$

Therefore,

$$
\left(\int_{\Omega}\left|u_{n}(t)\right|^{\frac{2^{*}}{2} q}\right)^{\frac{2}{2^{*}} \theta} \geq \frac{1}{\left\|u_{0}\right\|_{L^{1}(\Omega)}^{q(1-\theta)}} \int_{\Omega}\left|u_{n}(t)\right|^{q},
$$

where we have assumed that $u_{0} \not \equiv 0$ (otherwise there is nothing to prove). Inserting (2.15) into (2.13), we obtain

$$
\frac{d}{d t} \int_{\Omega}\left|u_{n}(t)\right|^{q}+A\left(\int_{\Omega}\left|u_{n}(t)\right|^{q}\right)^{\frac{1}{\theta}} \leq C_{3} q \int_{\Omega}\left|u_{n}(t)\right|^{q},
$$

where

$$
A=\frac{\alpha(q-1)}{q \mathcal{S}\left\|u_{0}\right\|_{L^{1}(\Omega)}^{\frac{q(1-\theta)}{\theta}}}
$$

Since

$$
\frac{1}{\theta}=1+\delta, \quad \text { with } \quad \delta=\frac{2}{N(q-1)},
$$

we thus have

$$
\frac{d}{d t} \int_{\Omega}\left|u_{n}(t)\right|^{q}+A\left(\int_{\Omega}\left|u_{n}(t)\right|^{q}\right)^{1+\delta} \leq C \int_{\Omega}\left|u_{n}(t)\right|^{q},
$$

where to simplify the notation we have set $C=C_{3} q$. Define now

$$
y(t)=\int_{\Omega}\left|u_{n}(t)\right|^{q} .
$$


From (2.16) we have that $y(t)$ is such that

$$
y^{\prime}(t)+A y(t)^{1+\delta} \leq C y(t)
$$

where $A$ and $C$ are as above. Multiplying by $\mathrm{e}^{-C t}$, we have

$$
\mathrm{e}^{-C t} y^{\prime}(t)-C \mathrm{e}^{-C t} y(t)+A \mathrm{e}^{-C t} y(t)^{1+\delta} \leq 0,
$$

that is,

$$
\left[\mathrm{e}^{-C t} y(t)\right]^{\prime}+A \mathrm{e}^{-C t} y(t)^{1+\delta} \leq 0 .
$$

Define $z(t)=\mathrm{e}^{-C t} y(t)$, so that we have

$$
z^{\prime}(t)+A \mathrm{e}^{-C t}\left[\mathrm{e}^{C t} z(t)\right]^{1+\delta} \leq 0,
$$

that is

$$
z^{\prime}(t)+A \mathrm{e}^{C \delta t} z(t)^{1+\delta} \leq 0
$$

Dividing by $z(t)^{1+\delta}$, we have

$$
\frac{z^{\prime}(t)}{z(t)^{1+\delta}}+A \mathrm{e}^{C \delta t} \leq 0
$$

which can be rewritten as

$$
\left[-\frac{1}{\delta z(t)^{\delta}}+\frac{A \mathrm{e}^{C \delta t}}{C \delta}\right]^{\prime} \leq 0
$$

so that the function

$$
t \mapsto \frac{A \mathrm{e}^{C \delta t}}{C \delta}-\frac{1}{\delta z(t)^{\delta}} \quad \text { is decreasing, }
$$

or, simplifying $\delta$, that the function

$$
t \mapsto A \mathrm{e}^{C \delta t}-C z(t)^{-\delta} \text { is decreasing, }
$$

Thanks to (2.17), we have that, for every $0<t<T$,

$$
A \mathrm{e}^{C \delta t}-C z(t)^{-\delta} \leq A-C z(0)^{-\delta} \leq A,
$$

and thus,

$$
z(t)^{-\delta} \geq A C^{-1}\left(\mathrm{e}^{C \delta t}-1\right) \quad \text { for every } t \in(0, T)
$$

that is,

$$
z(t) \leq \frac{1}{\left[A C^{-1}\left(\mathrm{e}^{C \delta t}-1\right)\right]^{\frac{1}{\delta}}} \quad \text { for every } t \in(0, T),
$$


Recalling the definition of $z(t)$, we thus have that

$$
y(t) \leq \frac{\mathrm{e}^{C t}}{\left[A C^{-1}\left(\mathrm{e}^{C \delta t}-1\right)\right]^{\frac{1}{\delta}}} \quad \text { for every } t \in(0, T) .
$$

that is

$$
\int_{\Omega}\left|u_{n}(t)\right|^{q} \leq \frac{\mathrm{e}^{C t}}{\left[A C^{-1}\left(\mathrm{e}^{C \delta t}-1\right)\right]^{\frac{1}{\delta}}} \quad \text { for every } t \in(0, T) .
$$

Hence, passing to the limit on $n$ we deduce that, for almost every $t$ in $(0, T)$ we have

$$
\int_{\Omega}|u(t)|^{q} \leq \frac{\mathrm{e}^{C t}}{\left[A C^{-1}\left(\mathrm{e}^{C \delta t}-1\right)\right]^{\frac{1}{\delta}}}=\frac{1}{\left(A C^{-1}\right)^{\frac{1}{\delta}}} \frac{\mathrm{e}^{C t}}{\left(\mathrm{e}^{C \delta t}-1\right)^{\frac{1}{\delta}}} .
$$

Recalling the value of $A$, we thus have

$$
\int_{\Omega}|u(t)|^{q} \leq C_{4}\left\|u_{0}\right\|_{L^{1}(\Omega)}^{\frac{q(1-\theta)}{\delta \theta}} \frac{\mathrm{e}^{C t}}{\left(\mathrm{e}^{C \delta t}-1\right)^{\frac{1}{\delta}}} \quad \text { for almost every } t \in(0, T),
$$

where $C_{4}=\left(\frac{q \mathcal{S} C}{\alpha(q-1)}\right)^{\frac{1}{\delta}}$. Since

$$
\delta=\frac{1}{\theta}-1=\frac{1-\theta}{\theta}=\frac{2}{N(q-1)},
$$

we finally have that, for almost every $t$ in $(0, T)$

$$
\int_{\Omega}|u(t)|^{q} \leq C_{4}\left\|u_{0}\right\|_{L^{1}(\Omega)}^{q} \frac{\mathrm{e}^{C t}}{\left(\mathrm{e}^{C \delta t}-1\right)^{\frac{1}{\delta}}} .
$$

Recalling that $\mathrm{e}^{C \delta t}-1 \geq C \delta t$ for every $t$ (since $t \mapsto \mathrm{e}^{C \delta t}$ is convex), we therefore have that

$$
\|u(t)\|_{L^{q}(\Omega)} \leq C_{1} \frac{\left\|u_{0}\right\|_{L^{1}(\Omega)}}{t^{\frac{1}{\delta q}}} \quad \text { for almost every } t \text { in }(0, T),
$$

where

$$
c_{1}=\left[C_{4} \frac{\mathrm{e}^{C T}}{(C \delta)^{\frac{1}{\delta}}}\right]^{\frac{1}{q}}
$$

and hence (2.4) is proved.

To conclude the proof we observe that by (2.19) it follows that even if $u_{0} \in L^{1}(\Omega)$ it results

$u \in L^{\infty}\left(t_{0}, T ; L^{q}(\Omega)\right)$ for every $t_{0}$ in $(0, T)$, and for every $1<q<+\infty .(2.22)$ 
Moreover, it results

$$
\|u\|_{L^{\infty}\left(t_{0}, T ; L^{q}(\Omega)\right)} \leq c_{0}\left\|u_{0}\right\|_{L^{1}(\Omega)},
$$

where $c_{0}$ is a constant depending only on $t_{0}, q, N, \alpha, T$ and $B$.

Finally, using the previous regularity in (2.12) we deduce that for every $q \geq 2$ $\left.|\nabla| u\right|^{\frac{q}{2}-1} u\left|\in L^{2}\left(t_{0}, T ; L^{2}(\Omega)\right), \quad\right| \nabla u \mid \in L^{2}\left(t_{0}, T ; L^{2}(\Omega)\right)$ for every $t_{0}$ in $(0, T)$.

\subsection{Behaviour for $t$ large}

We show here that our problem admits a global solution $u$ (defined in all the set $\Omega \times(0,+\infty))$ and we study its behavior for $t$ large. To this aim, we recall that by a global solution of (1.1) we mean a function $u$ which solves (1.1) for every $T>0$.

Theorem 2.2. Assume (1.3) and that (1.2) and (1.5) hold true in $\Omega \times(0,+\infty)$. Let $u$ be the solution of (1.1) given by Theorem 2.1. Then $u$ can be extended to a global solution defined in $\Omega \times(0,+\infty)$ (that we denote again $u$ ) satisfying

$$
\begin{aligned}
& u \in L^{\infty}\left(t_{0},+\infty ; L^{q}(\Omega)\right) \text { for every } t_{0}>0, \text { and for every } 1<q<+\infty, \\
& \|u\|_{L^{\infty}\left(t_{0},+\infty ; L^{q}(\Omega)\right)} \leq\left\|u_{0}\right\|_{L^{1}(\Omega)},
\end{aligned}
$$

where $c_{0}$ is as in (2.3) a constant depending only on $t_{0}, q, N, \alpha$ and $B$, and such that for every $q \geq 2$ and $t_{0}>0$ we have

$$
\left.|\nabla| u\right|^{\frac{q}{2}-1} u\left|\in L_{\mathrm{loc}}^{2}\left(t_{0},+\infty ; L^{2}(\Omega)\right), \quad\right| \nabla u \mid \in L_{\mathrm{loc}}^{2}\left(t_{0},+\infty ; L^{2}(\Omega)\right) .
$$

Moreover, if for some $t_{0}>0$ it results

$$
|E(x, t)| \leq b(t)|B(x)|, \quad \text { where } b \in L^{2}\left(t_{0},+\infty\right) \text { and } B(x) \in L^{N}(\Omega),
$$

or more generally, if

$$
|E(x, t)| \in L^{2}\left(t_{0},+\infty ; L^{r}(\Omega)\right), \text { for somer }>2,
$$

then

$$
\lim _{t \rightarrow+\infty}\|u(t)\|_{L^{q}(\Omega)}=0 \quad \text { for every } 1<q<+\infty .
$$

Proof. Let $T>0$ be arbitrarily fixed and let $u$ be the solution of (1.1) given by Theorem 2.1. Hence $u$ is obtained as the almost everywhere limit in $Q_{T}$ of a sequence $u_{n}$ in $L^{2}\left(0, T ; W_{0}^{1,2}(\Omega)\right) \cap C\left([0, T] ; L^{2}(\Omega)\right)$ of weak solutions of $(2.5)$. Notice that each $u_{n}$ can be extended to a global solution of (2.5), that we denote again $u_{n}$. Hence $u_{n}$ belongs to $L_{\text {loc }}^{2}\left([0,+\infty) ; W_{0}^{1,2}(\Omega)\right) \cap C_{\text {loc }}\left([0,+\infty) ; L^{2}(\Omega)\right)$, and solves $(2.5)$ in every set $Q_{\mathrm{T}}$ for every T $>0$ arbitrarily fixed. We show now that we can extend the 
solution $u$ of (1.1) in $Q_{T}$ to a global solution. To this aim let us denote with $u_{n}^{(1)}$ the subsequence of $u_{n}$ that converges almost everywhere in $Q_{2 T}$ to a weak solution $u^{(2)}$ of (1.1) in $Q_{2 T}$. By construction $u=u^{(2)}$ in $Q_{T}$. Now, let us denote with $u_{n}^{(2)}$ the subsequence of $u_{n}^{(1)}$ that converges almost everywhere in $Q_{3 T}$ to a weak solution $u^{(3)}$ of (1.1) in $Q_{3 T}$. By construction $u^{(2)}=u^{(3)}$ in $Q_{2 T}$. Iterating this procedure, the function $u(x, t) \equiv u^{(n)}(x, t)$ in $Q_{n T}$ (for every integer $n$ ) is well defined and is a global solution of (1.1) which, by construction and thanks to Theorem 2.1, satisfies (2.24)-(2.26).

We show now that the global solution $u$ constructed above, under further assumptions on $E$, satisfies the other estimates enounced in Theorem 2.2.

To this aim, proceeding as in the proof of (2.8), and integrating in time, we deduce that for every $0<t_{0}<t_{1}<t_{2}$

$$
\begin{gathered}
\int_{\Omega}\left|u_{n}\left(t_{2}\right)\right|^{q}-\int_{\Omega}\left|u_{n}\left(t_{1}\right)\right|^{q}+\frac{q \alpha(q-1)}{2} \int_{t_{1}}^{t_{2}} \int_{\Omega}\left|\nabla u_{n}(t)\right|^{2}\left|u_{n}(t)\right|^{q-2} \\
\leq \frac{q(q-1)}{2 \alpha} \int_{t_{1}}^{t_{2}} \int_{\Omega}|E(x, t)|^{2}\left|u_{n}(t)\right|^{q} .
\end{gathered}
$$

Now, thanks to the Poincaré inequality we deduce

$$
\begin{gathered}
\int_{\Omega}\left|u_{n}\left(t_{2}\right)\right|^{q}-\int_{\Omega}\left|u_{n}\left(t_{1}\right)\right|^{q}+\frac{2 \alpha(q-1) C_{P}}{q} \int_{t_{1}}^{t_{2}} \int_{\Omega}\left|u_{n}(t)\right|^{q} \\
\leq \frac{q(q-1)}{2 \alpha} \int_{t_{1}}^{t_{2}} \int_{\Omega}|E(x, t)|^{2}\left|u_{n}(t)\right|^{q},
\end{gathered}
$$

where $C_{P}$ is the Poincaré constant. We can rewrite the previous estimate in the following way

$$
\int_{\Omega}\left|u_{n}\left(t_{2}\right)\right|^{q}-\int_{\Omega}\left|u_{n}\left(t_{1}\right)\right|^{q}+M \int_{t_{1}}^{t_{2}} \int_{\Omega}\left|u_{n}(t)\right|^{q} \leq \int_{t_{1}}^{t_{2}} g_{n}(t) d t .
$$

where we have set

$$
M=\frac{2 \alpha(q-1) C_{P}}{q} \text { and } g_{n}(t)=\frac{q(q-1)}{2 \alpha} \int_{\Omega}|E(x, t)|^{2}\left|u_{n}(t)\right|^{q} .
$$

Applying Proposition 3.2 in [11] we obtain

$$
\int_{\Omega}\left|u_{n}(t)\right|^{q} \leq \int_{\Omega}\left|u_{n}\left(t_{0}\right)\right|^{q} \mathrm{e}^{-M t}+\int_{t_{0}}^{t} g_{n}(s) d s,
$$

and if $g_{n} \in L^{1}\left(t_{0},+\infty\right)$ (again by Proposition 3.2) we get for every $t>t_{0}$

$$
\int_{\Omega}\left|u_{n}(t)\right|^{q} \leq \Lambda_{n} \mathrm{e}^{-\frac{M}{2} t}+\int_{\frac{t}{2}}^{t} g_{n}(s) d s
$$

where

$$
\Lambda_{n}=\int_{\Omega}\left|u_{n}\left(t_{0}\right)\right|^{q}+\left\|g_{n}\right\|_{L^{1}\left(t_{0},+\infty\right)}
$$


Notice that thanks to (2.25) it results

$$
\Lambda_{n} \leq \Lambda_{1} \equiv c_{0}^{q}\left\|u_{0}\right\|_{L^{1}(\Omega)}^{q}+\left\|g_{n}\right\|_{L^{1}\left(t_{0},+\infty\right)} .
$$

Suppose now that

$$
|E(x, t)| \leq b(t)|B(x)|, \quad b \in L^{2}\left(t_{0},+\infty\right) .
$$

Then

$$
\begin{aligned}
& \left\|g_{n}\right\|_{L^{1}\left(t_{0},+\infty\right)} \leq c \int_{t_{0}}^{+\infty} \int_{\Omega} b(t)^{2}|B(x)|^{2}\left|u_{n}\right|^{q} \\
& \leq c \int_{t_{0}}^{+\infty} \int_{\Omega} b^{2}\left[|B(x)|^{N}+\left|u_{n}\right|^{\frac{N q}{N-2}}\right] \\
& \leq c\left(\int_{t_{0}}^{+\infty} b^{2}\right)\left[\int_{\Omega}|B|^{N}+\left\|u_{n}\right\|^{\frac{N q}{N-2}} L_{L^{\infty} t_{0},+\infty ; L^{\left.\frac{N q}{N-2}(\Omega)\right)}}^{N-2}\right] \\
& \leq C\left(\int_{t_{0}}^{+\infty} b^{2}\right)\left[\int_{\Omega}|B|^{N}+\left\|u_{0}\right\|_{L^{1}(\Omega)}^{\frac{N q}{N-2}}\right],
\end{aligned}
$$

and

$$
\int_{\frac{t}{2}}^{t} g_{n}(s) d s \leq C\left(\int_{\frac{t}{2}}^{t} b^{2}\right)\left[\int_{\Omega}|B|^{N}+\left\|u_{0}\right\|_{L^{1}(\Omega)}^{\frac{N q}{N-2}}\right] .
$$

If instead one assumes that

$$
E \in L^{2}\left(t_{0},+\infty ; L^{r}(\Omega)\right), \quad \text { for some } r>2,
$$

then it results

$$
\begin{aligned}
& \left\|g_{n}\right\|_{L^{1}\left(t_{0},+\infty\right)} \leq c \int_{t_{0}}^{+\infty} \int_{\Omega}|E(x, t)|^{2}\left|u_{n}\right|^{q} \leq c \int_{t_{0}}^{+\infty}\left(\int_{\Omega}|E(x, t)|^{r}\right)^{\frac{2}{r}}\left(\int_{\Omega}\left|u_{n}\right|^{\frac{q r}{r-2}}\right)^{1-\frac{2}{r}} \\
& \leq c\left[\int_{t_{0}}^{+\infty}\left(\int_{\Omega}|E(x, t)|^{r}\right)^{\frac{2}{r}}\right]\left\|u_{n}\right\|_{L^{\infty}\left(t_{0},+\infty ; L^{\frac{q r}{r-2}}(\Omega)\right)}^{q} \\
& \leq C\left[\int_{t_{0}}^{+\infty}\left(\int_{\Omega}|E(x, t)|^{r}\right)^{\frac{2}{r}}\right]\left\|u_{0}\right\|_{L^{1}(\Omega)}^{q},
\end{aligned}
$$

and

$$
\int_{\frac{t}{2}}^{t} g_{n}(s) d s \leq C\left[\int_{\frac{t}{2}}^{t}\left(\int_{\Omega}|E(x, t)|^{r}\right)^{\frac{2}{r}}\right]\left\|u_{0}\right\|_{L^{1}(\Omega)}^{q} .
$$

Under either assumption (2.35) or (2.36) one therefore has that $\left\|g_{n}\right\|_{L^{1}\left(t_{0},+\infty\right)} \leq C, \quad \lim _{t \rightarrow+\infty} \int_{\frac{t}{2}}^{t} g_{n}(s) d s=0, \quad$ uniformly with respect to $n$. 
Thus,

$$
\lim _{t \rightarrow+\infty} \int_{\Omega}\left|u_{n}(t)\right|^{q}=0 \quad \text { uniformly with respect to } n,
$$

so that (recalling that the solution $u$ of (1.1) is the limit of $u_{n}$ )

$$
\lim _{t \rightarrow+\infty} \int_{\Omega}|u(t)|^{q}=0 .
$$

\section{3. $L^{\infty}$-regularity}

In this section we prove the following result.

Theorem 3.1. Assume (1.2), (1.3), (1.5) and

$$
|E| \in L^{s}\left(0, T ; L^{r}(\Omega)\right) \quad \text { with } r>2 \text { and } s \geq 1 \text { such that } \frac{N}{2 r}+\frac{1}{s}<\frac{1}{2} .
$$

Then there exists a solution u of (1.1) satisfying

$$
u \in L^{\infty}\left(\Omega \times\left(t_{0}, T\right)\right) \text { for every } t_{0} \in(0, T) .
$$

Moreover,

$$
\|u(t)\|_{L^{\infty}(\Omega)} \leq c \frac{\left\|u_{0}\right\|_{L^{1}(\Omega)}}{t^{\frac{N}{2}}}, \text { for every } t \in(0, T),
$$

where $c$ depends only on $\alpha, \beta, N, T$ and $E$.

Finally, if (1.2) and (1.5) hold true in $\Omega \times(0,+\infty)$ then $u$ can be extended to a global solution (that we denote again $u$ ) defined in all $\Omega \times(0,+\infty)$; this extension coincides with the one given by Theorem 2.2 ; if $|E|$ belongs to $L_{\mathrm{loc}}^{s}\left(0,+\infty ; L^{r}(\Omega)\right)$ and (2.37) holds true (for example if (2.35) or (2.36) holds true) then it results

$$
\lim _{t \rightarrow+\infty}\|u(t)\|_{L^{\infty}(\Omega)}=0 .
$$

Proof. We recall that under the assumptions of Theorem 2.1 (see Sect. 2.1) there exists a solution $u$ of (1.1) satisfying the regularity property (2.2) and estimate (2.3). Hence, if we assume that

$$
\left.|E| \in L^{s}\left(0, T ; L^{r}(\Omega)\right)\right) \text { with } r>2 \text { and } s \geq 1 \text { such that } \frac{N}{2 r}+\frac{1}{s}<\frac{1}{2},
$$

then there exist $\bar{s}>1$ and $\bar{r}$ (depending on $r$ and $s$ ) such that it results

$$
\left.|F| \stackrel{\text { def }}{=}|E(x, t) u| \in L^{\bar{s}}\left(t_{0}, T ; L^{\bar{r}}(\Omega)\right)\right) \quad \text { with } \bar{r}>2 \text { such that } \frac{N}{2 \bar{r}}+\frac{1}{\bar{s}}<\frac{1}{2} \text {, for every } t_{0}>0 \text {. }
$$


Notice that (3.5) is satisfied if for example $|E| \in L^{\infty}\left(0, T ; L^{r}(\Omega)\right)$ with $r>N$.

Thus, if we assume (3.5) we can apply Theorem 8.1 at page 192 of [10] to the solution $u$ of (1.1) constructed in Theorem 2.1 to conclude that

$$
u \in L^{\infty}\left(\Omega \times\left(t_{0}, T\right)\right) \text { for every } t_{0} \in(0, T) .
$$

Moreover it is also possible to estimate the $L^{\infty}$-norm of $u$ in dependence of the data and of the $L^{2}$-norm of $u$.

Now to complete the proof of the theorem we need to show the behavior of the $L^{\infty}$-norm of $u$ near zero and for $t$ large.

To this aim, we recall that applying Theorem 2 at page 18 of [1] we obtain the following estimate

$$
\|u\|_{L^{\infty}(Q(\rho))} \leq C\left[\rho^{-\frac{N+2}{2}}\|u\|_{L^{2}(Q(3 \rho))}+\rho^{\theta}\||u E|\|_{\bar{s}, \bar{r}, Q(3 \rho)}\right]
$$

where $C$ is a constant depending only on the data (i.e. on $\alpha, \beta, \theta$ and N) $Q(\rho)=R(\rho) \times$ $\left(t_{0}-\rho^{2}, t_{0}\right), R(\rho)$ is the open cube in $R^{N}$ of edge lenght $\rho$ centered in $x_{0} \in \Omega, \rho>0$ such that $Q(3 \rho) \subset Q_{T},\|\cdot\|_{s_{0}, r_{0}, Q(3 \rho)}$ denotes the norm $\|\cdot\|_{L^{s_{0}}\left(t_{0}-(3 \rho)^{2}, t_{0} ; L^{r_{0}}(K(3 \rho))\right)}$ and $\theta \in(0,1)$ is defined as follows

$$
\theta \leq 1-\left(\frac{N}{2 \bar{r}}+\frac{1}{\bar{s}}\right) \text { and } \theta \leq 1-\frac{1}{r} .
$$

We conclude the proof distinguishing the two cases of $t$ near zero and $t$ large.

Case 1: $t$ near zero. We observe that it results

$$
\|u\|_{L^{2}(Q(3 \rho))} \leq 3 \rho\|u\|_{L^{\infty}\left(t_{0}-(3 \rho)^{2}, t_{0} ; L^{2}(K(3 \rho))\right)}
$$

which implies

$$
\rho^{-\frac{N+2}{2}}\|u\|_{L^{2}(Q(3 \rho))} \leq 3 \rho^{-\frac{N}{2}}\|u\|_{L^{\infty}\left(t_{0}-(3 \rho)^{2}, t_{0} ; L^{2}(K(3 \rho))\right)}
$$

Hence, choosing $(3 \rho)^{2}=\frac{t_{0}}{2}$ and $t$ arbitrarily in $\left(\frac{17}{18} t_{0}, t_{0}\right)$ (hence $t=\theta_{0} t_{0}$ with $\left.\theta_{0} \in\left(\frac{17}{18}, 1\right)\right)$ and using estimate $(2.4)$ we deduce

$$
\rho^{-\frac{N+2}{2}}\|u\|_{L^{2}(Q(3 \rho))} \leq C t^{-\frac{N}{4}} \frac{\left\|u_{0}\right\|_{L^{1}(\Omega)}}{t^{\frac{N}{4}}}=C \frac{\left\|u_{0}\right\|_{L^{1}(\Omega)}}{t^{\frac{N}{2}}} .
$$

Here with $C$ or $c$ we denote positive constants, depending only on the data in the structure conditions and on $E$, which can vary from line to line. Notice that with such a choice of $\rho$ the left hand side of (3.8) is greater then $\|u(t)\|_{L^{\infty}(K(\rho))}$. Hence, it remains to estimate the last term in the right hand side of (3.8).

Let us consider the particular case when $|E| \in L^{\infty}\left(0, T ; L^{r}(\Omega)\right)$ with $r>N$ (i.e. (3.5) with $s=+\infty)$. Then $|u E| \in L^{\infty}\left(t_{0}, T ; L^{\bar{r}}(\Omega)\right)$ for every $N<\bar{r}<r$ for every $t_{0}>0$, and we have

$$
\||u E|\|_{\infty, \bar{r}, Q(3 \rho)} \leq\||E|\|_{\infty, r, Q(3 \rho)}\|u\|_{\infty, \frac{\bar{r} r}{r-\bar{r}}, Q(3 \rho)}
$$


Hence, by (3.13) and (2.4) we deduce that

$$
\rho^{\theta}\||u E|\|_{\bar{s}, \bar{r}, Q(3 \rho)} \leq c t^{\frac{\theta}{2}} \frac{\left\|u_{0}\right\|_{L^{1}(\Omega)}}{t^{\frac{N}{2}\left(1-\frac{r-\bar{r}}{\bar{r} r}\right)}}
$$

which implies, since $\theta>0$ and being possible any choice of $\bar{r}$ satisfying $N<\bar{r}<r$, the following estimate

$$
\rho^{\theta}\||u E|\|_{\bar{s}, \bar{r}, Q(3 \rho)} \leq c \frac{\left\|u_{0}\right\|_{L^{1}(\Omega)}}{t^{\frac{N}{2}}} .
$$

Now, by (3.15), (3.12) and (3.8) we can conclude that for $t$ in $(0, T)$ it results

$$
\|u(t)\|_{L^{\infty}(K(\rho))} \leq c \frac{\left\|u_{0}\right\|_{L^{1}(\Omega)}}{t^{\frac{N}{2}}},
$$

which implies, thanks to the arbitrariness of $K(\rho)$,

$$
\|u(t)\|_{L^{\infty}(\Omega)} \leq c \frac{\left\|u_{0}\right\|_{L^{1}(\Omega)}}{t^{\frac{N}{2}}} .
$$

We conclude the proof considering the general case when $|E|$ satisfies (3.5) with $s \neq+\infty$. In this case we estimate the last term in (3.8) as follows

$$
\begin{aligned}
& \rho^{\theta}\||u E|\|_{\bar{s}, \bar{r}, Q(3 \rho)} \leq \rho^{\theta}\||E|\|_{s, r, Q(3 \rho)}\||u|\|_{\frac{\bar{s} s}{s-\bar{s}}, \frac{\bar{r} r}{r-\bar{r}}, Q(3 \rho)} \\
& \quad \leq \rho^{\theta+2 \frac{s-\bar{s}}{\bar{s} s}}\||E|\|_{s, r, Q(3 \rho)}\||u|\|_{\infty, \frac{\bar{r} r}{r-\bar{r}}, Q(3 \rho)},
\end{aligned}
$$

for every $\bar{s}<s$ and $\bar{r}<r$ satisfying (3.6). Hence, by the previous estimate and (2.4) we deduce, for every $t$ in $(0, T)$, that

$$
\rho^{\theta}\||u E|\|_{\bar{s}, \bar{r}, Q(3 \rho)} \leq c t^{\frac{\theta}{2}+\frac{s-\bar{s}}{\bar{s} s}} \frac{\left\|u_{0}\right\|_{L^{1}(\Omega)}}{t^{\frac{N}{2}\left(1-\frac{r-\bar{r}}{\bar{r} r}\right)}}
$$

which implies (thanks to the arbitrariness of $\bar{s}<s$ and $\bar{r}<r$ ) that (3.15) holds true and hence estimate (3.16) is true also in this more general case.

Case 2: $t$ large. To study the solution for $t$ large we need to assume further structure assumptions to guarantee the existence of a solution defined for every value of $t$. Hence, let us assume that (1.2) and (1.5) hold true in $\Omega \times(0,+\infty)$. Thus, by Theorem $2.2 u$ can be extended to a global solution (that we denote again $u$ ) defined in all $\Omega \times(0,+\infty)$. Moreover, assuming also that $|E| \in L_{\text {loc }}^{s}\left(0,+\infty ; L^{r}(\Omega)\right)$ we obtain that estimate (3.8) holds true for every $Q(3 \rho) \subset \Omega \times(0,+\infty)$.

Hence, if (2.37) holds true (for example if (2.35) or (2.36) holds true) then by (3.8) we deduce that also

$$
\lim _{t \rightarrow+\infty}\|u(t)\|_{L^{\infty}(K(\rho))}=0
$$

and hence, thanks to the arbitrariness of $K(\rho)$, we conclude that

$$
\lim _{t \rightarrow+\infty}\|u(t)\|_{L^{\infty}(\Omega)}=0
$$




\section{4. $E$ does not belong to $L^{\infty}\left(0, T ; L^{N}(\Omega)\right)$}

We consider here a particular case when $E \notin L^{\infty}\left(0, T ; L^{N}(\Omega)\right)$. We have the following result.

Theorem 4.1. Assume that 0 belongs to $\Omega$, that (1.2), (1.3) hold, and that

$$
|E(x, t)| \leq \frac{\gamma}{|x|} \text { with } \gamma<\alpha(N-2)
$$

Then there exists a solution $u$ of (1.1) satisfying the following estimate for every $1 \leq q<(N-2) \frac{\alpha}{\gamma}$ and for a.e. $t \in(0, T)$

$$
\|u(t)\|_{L^{q}(\Omega)} \leq c \frac{\left\|u_{0}\right\|_{L^{1}(\Omega)}}{t^{\frac{N}{2} \frac{1}{q^{\prime}}}}
$$

where $c=c(N, q, \alpha, \gamma)$.

Proof. Proceeding as in the proof of Theorem 2.1 we deduce that (2.8) holds true with $B(x)=\frac{\gamma}{|x|}$, i.e.

$$
\frac{1}{q} \frac{d}{d t} \int_{\Omega}\left|u_{n}(t)\right|^{q}+\frac{\alpha(q-1)}{2} \int_{\Omega}\left|\nabla u_{n}(t)\right|^{2}\left|u_{n}(t)\right|^{q-2} \leq \frac{(q-1)}{2 \alpha} \gamma^{2} \int_{\Omega} \frac{\left|u_{n}(t)\right|^{q}}{|x|^{2}} .
$$

Recalling Hardy inequality, we thus have that

$$
\begin{aligned}
& \frac{1}{q} \frac{d}{d t} \int_{\Omega}\left|u_{n}(t)\right|^{q}+\frac{\alpha(q-1)}{2} \int_{\Omega}\left|\nabla u_{n}(t)\right|^{2}\left|u_{n}(t)\right|^{q-2} \\
& \quad \leq \frac{(q-1)}{2 \alpha} \frac{\gamma^{2}}{\mathcal{H}^{2}} \int_{\Omega}\left|\nabla\left[\left|u_{n}(t)\right|^{\frac{q}{2}-1} u_{n}\right]\right|^{2}=\frac{(q-1)}{2 \alpha} \frac{\gamma^{2} q^{2}}{4 \mathcal{H}^{2}} \int_{\Omega}\left|\nabla u_{n}(t)\right|^{2}\left|u_{n}(t)\right|^{q-2},
\end{aligned}
$$

that is

$$
\frac{1}{q} \frac{d}{d t} \int_{\Omega}\left|u_{n}(t)\right|^{q}+\frac{1}{2} \alpha(q-1)\left(1-\frac{1}{\alpha^{2}} \frac{\gamma^{2} q^{2}}{4 \mathcal{H}^{2}}\right) \int_{\Omega}\left|\nabla u_{n}(t)\right|^{2}\left|u_{n}(t)\right|^{q-2} \leq 0
$$

We observe that, since $\mathcal{H}=\frac{N-2}{2}$, it results

$$
\gamma q<2 \mathcal{H} \alpha \Longleftrightarrow q<(N-2) \frac{\alpha}{\gamma} \text {. }
$$

Since we need to have $q \geq 1$, by (4.4) we deduce that under the following assumption

$$
\gamma<\alpha(N-2)
$$

estimate (4.3) holds true for every $1 \leq q<(N-2) \frac{\alpha}{\gamma}$. By Sobolev inequality and (4.3) it follows for every $0<t_{1}<t_{2} \leq T$

$$
\int_{\Omega}\left|u_{n}\left(t_{2}\right)\right|^{q}-\int_{\Omega}\left|u_{n}\left(t_{1}\right)\right|^{q}+c_{1} \int_{t_{1}}^{t_{2}}\left\|u_{n}\right\|_{\frac{2^{*}}{2} q}^{q} \leq 0
$$


where $c_{1}=\frac{1}{2} \alpha(q-1)\left(1-\frac{1}{\alpha^{2}} \frac{\gamma^{2} q^{2}}{4 \mathcal{H}^{2}}\right) \frac{4}{q^{2} S}$. We recall that since $E \in L^{2}\left(Q_{T}\right)$ it results

$$
\left\|u_{n}(t)\right\|_{L^{1}(\Omega)} \leq\left\|u_{0}\right\|_{L^{1}(\Omega)}
$$

(see the proof of Lemma 3.1 in [4], or (2.1)). Hence, we can apply Theorem 2.1 in [13] (since the exponents in the integral inequality (4.6) satisfy all the needed requirements) and we can conclude that if (4.5) is satisfied then for every $1 \leq q<(N-2) \frac{\alpha}{\gamma}$ it results

$$
\left\|u_{n}(t)\right\|_{L^{q}(\Omega)} \leq c_{3} \frac{\left\|u_{0}\right\|_{L^{1}(\Omega)}}{t^{\frac{N}{2} \frac{1}{q^{\prime}}}},
$$

where $c_{3}=\left(\frac{N(q-1)}{2 c_{1}}\right)^{\frac{N}{2} \frac{1}{q^{\prime}}}$, from which the result follows (since the solution $u$ of (1.1) is the limit of $u_{n}$ ).

\section{Acknowledgements}

The authors would like to thank Google and its Google Meet software for supporting all the discussions (both preliminary and final) which generated the present paper during the COVID-19 lockdown in Italy.

Publisher's Note Springer Nature remains neutral with regard to jurisdictional claims in published maps and institutional affiliations.

\section{REFERENCES}

[1] D. G. Aronson, J. Serrin. Local behavior of solutions of quasilinear parabolic equations. Arch. Rational Mech. Anal., 25 (1967), 81-122.

[2] L. Boccardo. Some developments on Dirichlet problems with discontinuous coefficients. Boll. Unione Mat. Ital. (9), 2 (2009), 285-297.

[3] L. Boccardo, S. Buccheri, G. R. Cirmi. Two linear noncoercive Dirichlet problems in duality. Milan J. Math., 86 (2018), 97-104.

[4] L. Boccardo, L. Orsina, A. Porretta. Some noncoercive parabolic equations with lower order terms in divergence form. J. Evol. Equ., 3 (2003), 407-418.

[5] F. Cipriani, G. Grillo. Uniform bounds for solutions to quasilinear parabolic equations. J. Differential Equations, 177 (2001), 209-234.

[6] R.G. Cirmi, S. D'Asero, S. Leonardi, M.M. Porzio. Local regularity results for solutions of linear elliptic equations with drift term. Adv. Calc. Var. https://doi.org/10.1515/acv-2019-0048(to appear).

[7] T. Coulhon, D. Hauer. Functional inequalities and regularizing effect of nonlinear semigroupstheory and application. In: SMAI—Mathématiques et Applications (2020), pp. 1-195.

[8] T. Del Vecchio, M. R. Posteraro. Existence and regularity results for nonlinear elliptic equations with measure data. Adv. Differential Equations, 1 (1996), 899-917.

[9] F. Farroni, G. Moscariello. A nonlinear parabolic equation with drift term. Nonlinear Anal., 177 (2018), 397-412.

[10] O. A. Ladyženskaja, V. A. Solonnikov, N. N. Ural'ceva. Linear and quasilinear equations of parabolic type. Translations of the American Mathematical Society, American Mathematical Society, Providence (1968), xi+648.

[11] G. Moscariello, M. M. Porzio. Quantitative asymptotic estimates for evolution problems. Nonlinear Anal., 154 (2017), 225-240. 
[12] M. M. Porzio. On decay estimates. J. Evol. Equ., 9 (2009), 561-591.

[13] M. M. Porzio. On uniform and decay estimates for unbounded solutions of partial differential equations. J. Differential Equations, 259 (2015), 6960-7011.

[14] G. Stampacchia. Le problème de Dirichlet pour les équations elliptiques du second ordre à coefficients discontinus. Ann. Inst. Fourier (Grenoble), 15 (1965), 189-258.

[15] G. Stampacchia. Équations elliptiques du second ordre à coefficients discontinus. Les Presses de l'Université de Montréal, Montreal (1966), 326.

[16] L. Véron. Effets régularisants de semi-groupes non linéaires dans des espaces de Banach. Ann. Fac. Sci. Toulouse Math. (5), 1 (1979), 171-200.

\author{
Lucio Boccardo \\ Sapienza Università di Roma \\ P.le A. Moro 2 \\ 00185 Rome \\ Italy \\ E-mail:boccardo@mat.uniroma1.it \\ Luigi Orsina and Maria Michaela Porzio \\ Dipartimento di Matematica "G. Castelnuovo" \\ Sapienza Università di Roma \\ P.le A. Moro 2 \\ 00185 Rome \\ Italy \\ E-mail: orsina@mat.uniromal.it \\ Maria Michaela Porzio \\ E-mail: mariamichaela.porzio@uniromal.it
}

Accepted: 13 February 2021 